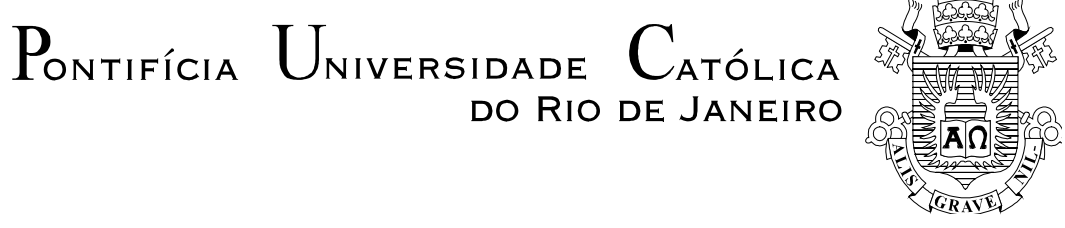

Rodrigo Otávio Santos von Doellinger

\title{
Gráfico de Controle Qui-Quadrado com Tamanho de Amostra Variável para Monitoramento de Perfis Lineares
}

Dissertação apresentada como requisito parcial para obtenção do grau de Mestre pelo Programa de Pósgraduação em Engenharia Elétrica do Departamento de Engenharia Elétrica da PUC-Rio.

Orientador: Prof. Reinaldo Castro Souza

Co-orientadora: Profa. Maysa Sacramento de Magalhães

Rio de Janeiro

Dezembro de 2010 


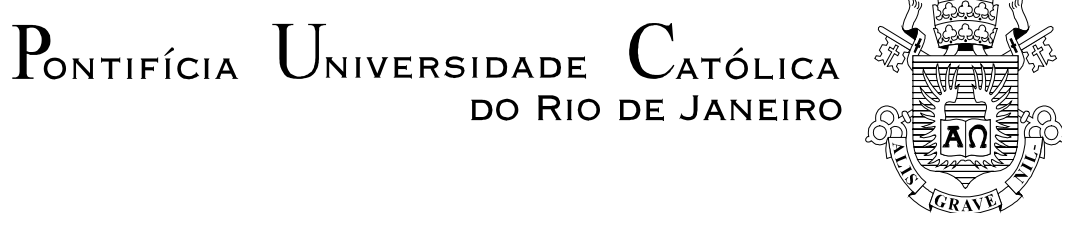

Rodrigo Otávio Santos von Doellinger

\begin{abstract}
Gráfico de Controle Qui-Quadrado com Tamanho de Amostra Variável para Monitoramento de Perfis Lineares
\end{abstract}

Dissertação apresentada como requisito parcial para obtenção do grau de Mestre pelo Programa de PósGraduação em Engenharia Elétrica do Departamento de Engenharia Elétrica do Centro Técnico Científico da PUC-Rio. Aprovada pela Comissão Examinadora abaixo assinada.

Prof. Reinaldo Castro Souza

Orientador

Departamento de Engenharia Elétrica - PUC-Rio

Profa. Maysa Sacramento de Magalhães

Co-Orientadora

Fundação Instituto Brasileiro de Geografia e Estatística

Prof. Antonio Fernando Branco Costa

Universidade Estadual Paulista Júlio de Mesquita Filho

Prof. Francisco Duarte Moura Neto

UERJ/IPRJ

Prof. José Eugenio Leal Coordenador Setorial do Centro

Técnico Científico 
Todos os direitos reservados. É proibida a reprodução total ou parcial do trabalho sem autorização da universidade, do autor e dos orientadores.

\section{Rodrigo Otávio Santos von Doellinger}

Graduou-se como Bacharel em Estatísticas na Escola Nacional de Ciências Estatísticas em 2005.

Ficha Catalográfica

Doellinger, Rodrigo Otávio Santos von

Gráfico de controle Qui-Quadrado com tamanho de amostra variável para monitoramento de perfis / Rodrigo Otávio Santos von Doellinger ; orientador: Reinaldo Castro Souza ; co-orientadora: Maysa Sacramento de Magalhães . - 2010.

77 f. ; $30 \mathrm{~cm}$

Dissertação (mestrado) - Pontifícia Universidade Católica do Rio de Janeiro, Departamento de Engenharia Elétrica, 2010.

Inclui bibliografia

1. Engenharia elétrica - Teses. 2. Controle estatístico de processos. 3. Gráficos de controle. 4. Perfil linear. 5. Regressão linear. 6. Cadeia de Markov. I. Souza, Reinaldo Castro. II. Maysa Sacramento de Magalhães III. Pontifícia Universidade Católica do Rio de Janeiro. Departamento de Engenharia Elétrica. IV. Título. 


\section{Agradecimentos}

Em primeiro lugar agradeço à minha querida mãe Maria Célia, que sempre esteve ao meu lado nos bons e nos maus momentos da minha vida.

Agradeço à minha noiva Vanessa dos Reis de Souza pela força e compreensão durante os três anos de mestrado.

Agradeço ao Professor Reinaldo Castro Souza pela atenção e pronto atendimento.

Meu especial agradecimento à Professora Maysa Sacramento de Magalhães pelo incentivo desde os tempos de graduação. Sua competência, dedicação e paciência foram fundamentais para a realização deste trabalho.

A todas as pessoas que me ajudaram durante o mestrado.

E, finalmente, a Deus por proporcionar-me a conclusão de mais uma etapa da vida. 


\section{Resumo}

von Doellinger, Rodrigo Otávio Santos; Souza, Reinaldo Castro (Orientador); de Magalhães, Maysa Sacramento (Co-orientadora). Gráfico de Controle Qui-Quadrado com Tamanho de Amostra Variável para Monitoramento de Perfis Lineares. Rio de Janeiro, 2010, 77p. Dissertação de Mestrado - Departamento de Engenharia Elétrica, Pontifícia Universidade Católica do Rio de Janeiro.

O monitoramento de perfis é utilizado para verificar a estabilidade de uma relação funcional envolvendo uma variável resposta e uma ou mais variáveis explicativas ao longo do tempo. Kang \& Albin (2000) fizeram uso do gráfico de controle qui-quadrado com parâmetros de projeto fixos para monitorar perfis lineares representados por um modelo de regressão linear simples. Nessa dissertação, com base nos estudos de Kang \& Albin (2000), desenvolvemos o gráfico de controle qui-quadrado com tamanho de amostra variável para o monitoramento de um perfil linear. O gráfico proposto monitora o intercepto e o coeficiente de inclinação de um modelo de regressão linear simples, com o uso de amostras com dois tamanhos. O desempenho do gráfico proposto é comparado com o desenvolvido por Kang \& Albin (2000). A medida de desempenho utilizada na comparação é o número médio de amostras até um sinal, obtida através de uma análise baseada em cadeias de Markov. Concluímos que é vantajoso utilizar o gráfico de controle qui-quadrado com tamanho de amostra variável.

\section{Palavras-chave}

Controle Estatístico de Processos; gráficos de controle; perfil linear; regressão linear; cadeia de Markov. 


\section{Abstract}

von Doellinger, Rodrigo Otávio Santos; Souza, Reinaldo Castro (Advisor); de Magalhães, Maysa Sacramento (Co-adivsor). Qui-Square Control Chart With Variable Sample Size to Monitor Linear Profiles. Rio de Janeiro, 2010, 77p. MSc Dissertation - Departamento de Engenharia Elétrica, Pontifícia Universidade Católica do Rio de Janeiro.

The monitoring of profiles is used to verify the stability of a functional relationship involving a response variable and one or more explanatory variables over time. Kang and Albin (2000) employed the chi-square control chart with fixed design parameters for monitoring linear profiles represented by a simple linear regression model. Based on the studies of Kang and Albin (2000), we developed the chi-square control chart with variable sample size for monitoring a linear profile. The proposed chart monitors the intercept and slope coefficient of a simple linear regression model, using two different sample sizes. The performance of the graph developed by Kang and Albin (2000) and the one presented here is compared. The average run length, obtained through a Markov chain, was used as performance measure to compare the two charts. We conclude that it is advantageous to use the chi-square control chart with variable sample size.

\section{Keywords}

Statistical Process Control; control charts; linear profile; linear regression; Markov chain. 


\section{Sumário}

1. Introdução 10

2. Trabalhos precedentes 15

3. Monitoramento de perfis lineares 23

3.1. Perfis como característicos de qualidade do processo 23

3.2. Modelo de regressão simples $\quad 30$

3.2.1. Estimação pelo método dos mínimos quadrados 33

3.3. A distribuição $\chi^{2} \quad 36$

3.4. Gráfico de controle $\chi^{2}$ com tamanho de amostra variável 38

3.4.1. Perfil linear monitorado 38

3.4.2. Estatística utilizada no monitoramento 39

3.5. Gráfico de controle $X^{2}$ com tamanho de amostra variável 40

4. Medida de desempenho do gráfico de controle
4.1. Cadeia de Markov

4.1.1. Processo fora de controle $\quad 43$

4.1.2. Probabilidades de transição 44

4.2. Obtenção do NMA 45

5. Análise de desempenho dos gráficos 47

6. Análise de sensibilidade do gráfico $\chi^{2}$ com tamanho de amostra
variável

6.1. Primeiro cenário $\quad 59$

6.2. Segundo cenário $\quad 61$

6.3. Comparação entre os dois cenários 62

$\begin{array}{ll}\text { 7. Conclusões } & 64\end{array}$

8. Referências bibliográficas $\quad 66$

$\begin{array}{ll}\text { 9. Apêndices } & 70\end{array}$ 


\section{Lista de tabelas}

Tabela 5.1 - NMA para os gráficos de controle TAV e PF com deslocamentos somente no intercepto, $\mathrm{n}_{0}=4$ e $\mathrm{n}_{1}=2$.

Tabela 5.2 - NMA para os gráficos de controle TAV e PF com deslocamentos somente no intercepto, $\mathrm{n}_{0}=4$ e $\mathrm{n}_{1}=3$.

Tabela 5.3 - NMA para os gráficos de controle TAV e PF com deslocamentos somente na inclinação, $\mathrm{n}_{0}=4$ e $\mathrm{n}_{1}=2$.

Tabela 5.4 - NMA para os gráficos de controle TAV e PF com deslocamentos somente na inclinação, $\mathrm{n}_{0}=4$ e $\mathrm{n}_{1}=3$.

Tabela 5.5 - NMA para os gráficos de controle TAV e PF com deslocamentos somente no desvio padrão.

Tabela 5.6 - Ganho percentual no cálculo de $E(N)$ para os gráficos de controle TAV e PF com deslocamentos somente no intercepto, $\mathrm{n}_{0}=4$ e $n_{1}=2$.

Tabela 5.7 - Ganho percentual no cálculo de $E(N)$ para os gráficos de controle TAV e PF com deslocamentos somente no intercepto, $\mathrm{n}_{0}=4$ e $n_{1}=3$.

Tabela 5.8 - Ganho percentual no cálculo de $E(N)$ para os gráficos de controle TAV e PF com deslocamentos somente na inclinação, $\mathrm{n}_{0}=4$ e $\mathrm{n}_{1}=2$.

Tabela 5.9 - Ganho percentual no cálculo de $E(N)$ para os gráficos de controle TAV e PF com deslocamentos somente na inclinação, $\mathrm{n}_{0}$ $=4$ e $n_{1}=3$.

Tabela 6.1 - Cálculo do NMA para o gráfico de controle com TAV com deslocamentos na inclinação e no intercepto, $\mathrm{n}_{0}=6$ e $\mathrm{n}_{1}=3$.

Tabela 6.2 - Cálculo do NMA para o gráfico de controle com TAV com deslocamentos variados no desvio-padrão, deslocamentos fixos de 0,025 na inclinação e 0,2 no intercepto, $n_{0}=6$ e $n_{1}=3$. 


\section{Lista de figuras}

Figura 5.1 - Ganho percentual entre os gráficos TAV e PF, em função de deslocamentos somente no intercepto, com $n_{0}=4$ e $n_{1}=2$.

Figura 5.2 - Ganho percentual entre os gráficos TAV e PF, em função de deslocamentos somente no intercepto, com $n_{0}=4$ e $n_{1}=3$.

Figura 5.3 - Ganho percentual entre os gráficos TAV e PF, em função de deslocamentos somente na inclinação, com $n_{0}=4$ e $n_{1}=2$.

Figura 5.4 - Ganho percentual entre os gráficos TAV e PF, em função de deslocamentos somente na inclinação, com $n_{0}=4$ e $n_{1}=3$.

Figura 6.1 - Evolução do NMA em função de $\mathrm{n}_{2}, \delta_{0}, \delta_{1}$ e com $\mathrm{n}_{0}=6$ e $\mathrm{n}_{1}=3$.

Figura 6.2 - Evolução do NMA em função de $\mathrm{n}_{2}, \gamma$ e com $\delta_{0}-0,2$, $\delta_{1}=0,025, \mathrm{n}_{0}=6$ e $\mathrm{n}_{1}=3$.

Figura 6.3 - Evolução do NMA em função do tamanho de amostra $\mathrm{n}_{2}$ para as duas situações em estudo. 